\title{
An Effective Wideband Spectrum Sensing Method for a Single CR
}

\author{
Ruiyan $\mathrm{Du}^{1,2, \mathrm{a}}$, Fulai Liu ${ }^{1,2, \mathrm{~b}}$, Zixuan Zhang ${ }^{1,2}$, Jinkuan Wang ${ }^{1,2}$ \\ ${ }^{1}$ Engineer Optimization \& Smart Antenna Institute, Northeastern University at Qinhuangdao, China \\ ${ }^{2}$ School of Information Science and Engineering, Northeastern University, Shenyang, China \\ aemail: ruiyandu@126.com, bemail: fulailiu@126.com
}

Keywords: cognitive radio; spectrum sensing; temporal smoothing; linear programming

\begin{abstract}
This paper presents a wideband spectrum sensing algorithm for a cognitive radio (CR) user equipped with a single receiving antenna. Firstly, the proposed method utilizes the temporal smoothing technique to form a virtual multi-antenna structure. Secondly, the wideband spectrum sensing problem is reformulated as a sparse reconstruction problem by exploiting a sparse representation of the virtual multi-antenna array covariance vector. Finally, the sparse reconstruction problem is modeled as a linear programming (LP) problem and hence can be solved efficiently. The presented method offers a number of advantages over other recently proposed methods. For examples, the unknown noise variances can be eliminated effectively by a linear transformation. It is computationally simpler since it is efficiently formulated in terms of the LP problem, etc. Simulation results are presented to verify the efficiency of the proposed method.
\end{abstract}

\section{Introduction}

For future CR networks, wideband or multi-band spectrum sensing is a crucial requirement for monitoring the primary users (PUs) activities and detecting spectrum holes for dynamic spectrum access, which can potentially improve spectrum utilization by allowing a secondary user (SU) or SUs to opportunistically utilize the spectrum if the primary user (PU) or PUs are inactive [1][2].

Various wideband spectrum sensing methods have been proposed in the literatures. For examples, ED is applied to wideband spectrum sensing problem [3]. A wavelet transform based method is proposed [4]. A simple approach to wideband spectrum sensing is presented [5]. Compressive sensing theory is applied in sense wideband spectrum [6]. A mixed-signal parallel segmented compressive sensing architecture is introduced for wideband spectrum sensing [7]. A distributed compressive sensing-based wideband sensing algorithm is presented for cooperative multihop CR networks [8]. Another framework of cooperative spectrum sensing is presented [9]. A multistage wiener filter (MSWF) based wideband spectrum sensing method is proposed [10].

In this paper, a new wideband spectrum sensing method is developed for a CR user equipped with a single receiving antenna. The proposed approach gives an effective sparse representation method by exploiting the virtual multi-antenna array covariance. Thus, all spectral holes can be effectively detected by finding the sparse coefficients. And then, the wideband spectrum sensing problem is modeled as a linear programming (LP) problem based on real-valued computation so that it can be solved efficiently.

The outline of the paper is organized as follows. The data model is described in Section 2. Section 3 introduces the proposed LPWS algorithm. Section 4 shows some simulation results. Finally, the conclusion is given in Section 5.

\section{Data Model}

Suppose that a total of $\mathrm{B} \mathrm{Hz}$ in the frequency range $\left[f_{L}, f_{H}\right]$ is available for a wideband $\mathrm{CR}$ network. The entire wideband channel can be divided into $K$ non-overlapping narrowband subbands. In a particular geographical region and within a particular time interval, some of the $K$ subbands might not be used by the primary users and are available for opportunistic spectrum access. The received signal of a secondary user can be given by 


$$
r(t)=\sum_{k=1}^{k_{0}} a_{k} S_{k}(t) e^{j 2 \pi f_{k} t}+w(t)
$$

where $t$ is the time index. $k_{0}$ denotes the unknown number of primary signals. $a_{k}$ stands for the amplitude of the $k$ th primary signal. $s_{k}(t)$ is the baseband representation. $f_{k}$ represents the carrier frequency of the $k$ th primary signal and is the center frequency of one of the occupied subbands.

Assume $P$ is the sampling rate, which is much higher than the data rate of the primary signal. The data samples at the CR receiver can be written as

$$
r(n)=\sum_{k=1}^{k_{0}} a_{k} s_{k}\left(\frac{n}{P}\right) e^{j \frac{2 \pi}{P} f_{k} n}+w\left(\frac{n}{P}\right) \quad(n=1,2, \cdots, N)
$$

where $N$ is the number of samples. In the remainder of the paper, unless it is necessary to write it explicitly, the amplitude $a_{k}$ in the data model is absorbed by $s_{k}(t)$, in which case, the amplitude of the primary signal is equal to $a_{k}$ instead of 1 . Then we can express (1) into vector form as

$$
r(n)=\phi(n) s(n)+w\left(\frac{n}{P}\right) \quad(n=1,2, \cdots, N)
$$

where $\phi(n)=\left[\phi_{1}^{n}, \cdots, \phi_{k_{0}}^{n}\right]$ with $\phi_{k}=\exp \left\{j(2 \pi / P) f_{k}\right\} . \quad s(n)=\left[s_{1}(n / P), \cdots, s_{k_{0}}(n / P)\right]^{T}$ in which $(\cdot)^{T}$ denotes the transposition.

\section{Algorithm Formulation}

First, we implement the temporal smoothing technique to form a virtual multi-antenna structure to get the covariance matrix.

\section{Virtual Multiple Antennas}

In the data model, $N$ samples of the signal are collected by a single-antenna receiver. We adopt a data stacking technique named temporal smoothing to form a virtual multi-antenna structure for the received data model. An $M$-factor temporal smoothed data matrix $\boldsymbol{Y}$ is constructed by stacking $M$ temporally shifted versions of the original data samples. As a result, $\boldsymbol{Y}$ will have a virtual multi-antenna structure. On the basis of (2), $\boldsymbol{Y}$ can be given as

$$
\boldsymbol{Y}=\left[\begin{array}{cccc}
r(1) & r(2) & \cdots & r(N-M+1) \\
r(2) & r(3) & \cdots & r(N-M+2) \\
\vdots & \vdots & \ddots & \vdots \\
r(M) & r(M+1) & \cdots & r(N)
\end{array}\right]+W
$$

where $\boldsymbol{W} \in C^{M, N-M+1}$ represents the noise term constructed from $w(n)$ in a similar way as $\boldsymbol{Y}$ is obtained from $r(n)$.

Assume that the primary signal $s_{k}(t)\left(k=1, \cdots, k_{0}\right)$ is narrow band, i.e., $s_{k}(t) \approx s_{k}(t+1 / P) \approx \cdots \approx s_{k}(t+M / P)$.In this case, all the block rows in the right-hand term of (3) are approximately equal, which means that $\boldsymbol{Y}$ has the following factorization

$$
\boldsymbol{Y} \approx \boldsymbol{A F}_{\mathrm{s}}+\boldsymbol{W} \in C^{M, N-M+1}
$$

where $\boldsymbol{A}=\left[a\left(\phi_{1}\right), \cdots, a\left(\phi_{k_{0}}\right)\right]$ with $a\left(\phi_{k}\right)=\left[\phi_{k}, \cdots, \phi_{k}^{M}\right]^{T} . \quad \boldsymbol{F}_{s}=[s(1) s(2) \cdots s(N-M+1)] \in c^{1, N-M+1}$ is a matrix collecting $N-M+1$ samples of the primary signals. The vector $s(k)$ is defined by (2).

Let $\boldsymbol{R}$ be the covariance matrix of the received signal data model which has a virtual multi-antenna structure, that is, $\boldsymbol{R}=\boldsymbol{Y} \boldsymbol{Y}^{H} /(N-M+1)$ where $(\cdot)^{H}$ denotes Hermitian transposition. 


\section{Sparse Representation}

Assume that all primary signals are uncorrelated each other. The noise is a complex Gaussian random process with zero-mean and uncorrelated with each of primary signals. Thus, the corresponding population covariance matrix $\boldsymbol{R}=\boldsymbol{A R}_{s} \boldsymbol{A}^{H}+\sigma_{n}^{2} \boldsymbol{I}_{M}$, where $\boldsymbol{R}_{s}=$ $\left(\boldsymbol{F}_{s} \boldsymbol{F}_{s}^{H}\right) /(N-M+1)=\operatorname{diag}\left(\boldsymbol{p}_{s}\right)$ in which $\boldsymbol{p}_{s}=\left(\sigma_{s_{1}}^{2}, \cdots, \sigma_{s_{k_{0}}}^{2}\right)^{T}$ with $\sigma_{s_{k}}^{2}$ denoting the signal power of the $k$ th primary signal source $s_{k}(t) . \sigma_{n}^{2}$ stands for the noise power. $\boldsymbol{I}_{M}$ denotes an $M \times M$ identity matrix. Let $\boldsymbol{z} \square \operatorname{vec}(\boldsymbol{R})=\left(A^{*} \square A\right) \boldsymbol{p}_{s}+\sigma_{n}^{2} \boldsymbol{1}_{M^{2}}$, where $\mathbf{1}_{M^{2}}=\left[\boldsymbol{e}_{1}^{T}, \boldsymbol{e}_{2}^{T}, \cdots \boldsymbol{e}_{M}^{T}\right]$ with $\boldsymbol{e}_{k}$ being a column vector of all zeros except a 1 at the $k$ th position. $\boldsymbol{A}^{*} \square \boldsymbol{A}=\left[\boldsymbol{a}^{*}\left(\phi_{1}\right) \otimes \boldsymbol{a}\left(\phi_{1}\right), \cdots, \boldsymbol{a}^{*}\left(\phi_{k_{0}}\right) \otimes \boldsymbol{a}\left(\phi_{k_{0}}\right)\right]$ in which $(\cdot)^{*}, \square$, and $\otimes$ represent the complex conjugate, Khatri-Rao product and Kronecker product, respectively. Let us construct a new matrix $\boldsymbol{A}_{1}$ of size $2 M-1$ from $\boldsymbol{A}^{*} \square \boldsymbol{A}$ where we have removed the repeated rows and also sorted them so that $\boldsymbol{A}_{1}=\left[\boldsymbol{a}_{1}\left(\phi_{1}\right), \boldsymbol{a}_{1}\left(\phi_{2}\right), \cdots, \boldsymbol{a}_{1}\left(\phi_{k_{0}}\right)\right]$ with $\boldsymbol{a}_{1}\left(\phi_{k}\right)=\left[\phi_{k}^{(M-1)}, \cdots, \phi_{k}, 1, \phi_{k}^{-1}, \cdots, \phi_{k}^{-(M-1)}\right]^{T}$. This is equivalent to removing the corresponding rows from the observation vector $\mathbf{z}$ and sorting them to get a new vector $z_{1}$ given by $z_{1}=A_{1} p_{s}+\sigma_{n}^{2} \boldsymbol{\eta}$, where $\boldsymbol{\eta}$ is a vector of all zeros except for a 1 at the $M$ th position. In order to eliminate the unknown noise variance $\sigma_{n}^{2}$, we can remove the element of $\boldsymbol{z}_{1}$ corresponding to the position of $\sigma_{n}^{2}$ in $\sigma_{n}^{2} \boldsymbol{\eta}$. Thus, we can obtain a new observation vector $\mathbf{z}_{2}=\boldsymbol{A}_{2} p_{s}+\sigma_{n}^{2} \eta$, where $\boldsymbol{A}_{2}=\boldsymbol{J} \boldsymbol{A}_{1}$ with $\boldsymbol{J}=\left[e_{1}^{T}, \cdots, e_{M-1}^{T}, e_{M+1}^{T}, \quad, \cdots, e_{2 M-1}^{T}\right]^{T}$ in which $\boldsymbol{e}_{m}(m \in[1,2 M-1]$ and $m \neq M)$ being a $1 \times(2 M-1)$ row vector with 1 at the $m$ th position and 0 elsewhere. $\boldsymbol{A}_{2}$ is the new steering matrix, i.e., $\boldsymbol{A}_{2}=\left[\boldsymbol{a}_{2}\left(\phi_{1}\right), \cdots, \boldsymbol{a}_{2}\left(\phi_{k_{0}}\right)\right]$ with $\boldsymbol{a}_{2}\left(\phi_{k}\right)=\left[\phi_{k}^{(M-1)}, \cdots, \phi_{k}, \phi_{k}^{-1}, \cdots, \phi_{k}^{-(M-1)}\right]^{T}$. This elimination operation avoids the estimation of noise variances.

Let $\left\{\hat{f}_{1}, \cdots, \hat{f}_{K}\right\}$ be a sampling grid of the center frequencies of $K$ non-overlapping narrowband subbands. If the $i$ th subband is occupied, $\hat{f}_{i}$ would be contained in the frequency components of the received signal. We construct an $N \times K$ matrix composed of steering vectors corresponding to the center frequency of each subband as its columns $\boldsymbol{A}_{2}(\phi)=\left[a_{2}\left(\hat{\phi}_{1}\right), \cdots, a_{2}\left(\hat{\phi}_{K}\right)\right]$. In this framework $\boldsymbol{A}_{2}(\phi)$ is known and does not depend on the actual channel occupancy state. We represent the signal power by a $K \times 1$ vector $\boldsymbol{x}$, where the element $x_{i}$ is nonzero and equals to $p_{i}$ if the $i$ th subband is occupied for some $i$ and zero otherwise. Thus, the signal model can be reduced to $\mathbf{z}_{2}=\boldsymbol{A}_{2}(\phi) \boldsymbol{x}$. Because the number of occupied subbands is small, the unknown power vector $\mathrm{x}$ is sparse, with only small number of nonzero elements.

\section{Solution Based on Linear Programming}

As just described previously, the wideband spectrum sensing problem can be cast as a problem of finding the sparsest solution of underdetermined linear system $\boldsymbol{z}_{2}=\boldsymbol{A}_{2}(\phi) \boldsymbol{x}$. Naturally, we should choose the $l_{0}$-norm as an ideal measure of sparsity. However, the $l_{0}$-norm minimization problem is nonconvex, NP-hard and thereby cannot be solved. Therefore, we can relax this problem to a simple $l_{1}$-norm minimization problem, where we equivalently seek to

$$
\min _{x} 1^{T} x \quad \text { s.t. } \quad z_{2}=A_{2}(\phi) x, \quad x \geq 0
$$

where 1 and $\mathbf{0}$ are $K \times 1$ vectors composed of 1 and 0 , respectively.

\section{Simulation Results}

Consider a wideband partitioned into $K=40$ non-overlapping subbands with equal bandwidth. 
The center frequency of each subband is from $1 \mathrm{MHz}$ to $40 \mathrm{MHz}$ with $1 \mathrm{MHz}$ intervals. The wideband channel is occupied by $k_{0}$ PUs, each user employs BPSK and the carrier frequency are randomly generated between 1-40 MHz. The Nyquist sampling rate $f_{s}$ is set to be $80 \mathrm{MHz} . N$ samples are used to collect transmitted signal at Nyquist sampling rate. The signal-to-noise-ratio (SNR) of a CR receiver is defined as the ratio of the average received signal power to noise power over the entire wideband.

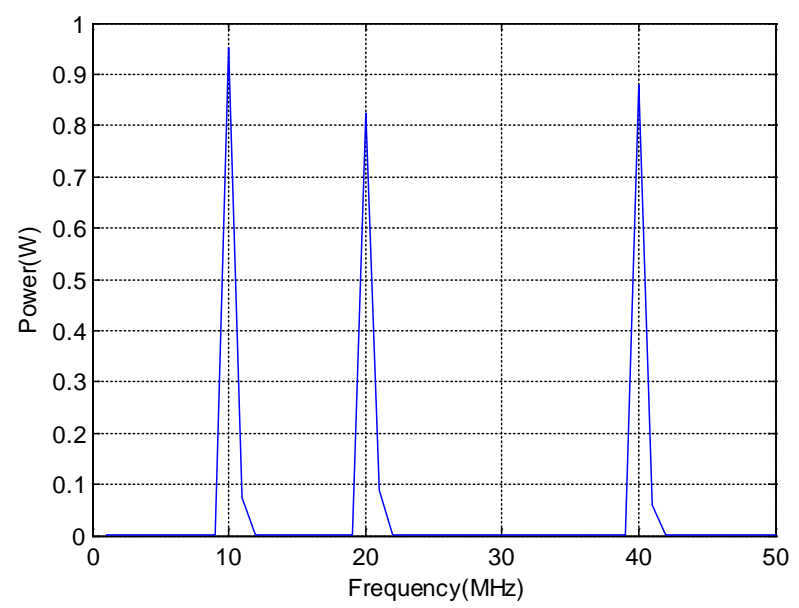

Fig.1. Reconstructed signal power versus frequency

In the first simulation, the wideband channel is occupied by three PUs and the carrier frequencies of PU signals are set to be $5 \mathrm{MHz}, 15 \mathrm{MHz}$ and $30 \mathrm{MHz}$, respectively. Fig. 1 depicts the reconstructed signal power vector $\boldsymbol{x}$ by solving the problem (6), for $N=256, M=5$, and SNR $=-10 \mathrm{~dB}$. It can be seen that the proposed method can reconstruct the signal power successfully. Since the occupied subbands locations correspond to the locations of the peaks in $\boldsymbol{x}$, three subbands can be decided to be occupied. In this way, spectral holes over a wideband channel can be detected effectively.

In the second simulation, we demonstrate the detection performance of the proposed algorithm with the number of active PUs changed. Fig.2 shows the probability of detection curves for different numbers of PUs with SNRs varying from -20dB to 0dB. $N$ and $M$ are set to be 256 and 6, respectively. It is shown that the probability of detection drops as the number of PUs increases, for the same SNR.

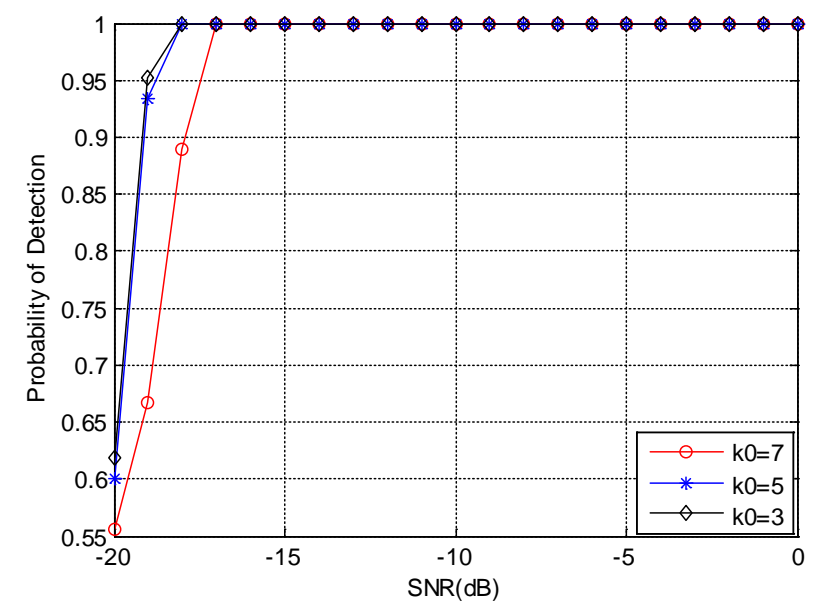

Fig.2 Probability of detection curves of the proposed algorithm

\section{Conclusion}

This paper presents a new wideband spectrum sensing algorithm. The wideband spectrum sensing problem can be transformed to the problem of estimation of signal power vector through sparse signal representation. Then spectrum holes can be detected effectively over a wideband 
channel by searching peaks in the reconstructed signal power. Furthermore, the sparse signal reconstruction problem can be modeled as a linear programming problem so that the resulting optimization problem can be solved efficiently. Simulation results show that the proposed algorithm can achieve high probability of detection in low SNR cases.

\section{Acknowledgement}

This work has been supported by the Program for New Century Excellent Talents in University (NCET-13-0105), and by the Support Program for Hundreds of Outstanding Innovative Talents in Higher Education Institutions of Hebei Province, under Grant No.BR2-259, and by the Fundamental Research Funds for the Central Universities under Grant No. N 130323001, and by the Program for Liaoning Excellent Talents in University (LJQ2012022), and by Directive Plan of Science Research from the Bureau of Education of Hebei Province, China, under Grant No.Z2011129, and by the National Natural Science Foundation of China under Grant No.60904035, and by the Specialized Research Fund for the Doctoral Program of Higher Education of China (No.20130042110003), and by the Doctor Foundation of Northeastern University at Qinhuangdao XNB201303.

\section{References}

[1] Sun H. J., Chen Y. F. Wideband spectrum sensing for cognitive radio networks: a survey [J]. IEEE Wireless Communications, 2013, 20(2): 74-81.

[2] Sun, Z. W., Laneman L. N. Performance metrics, sampling schemes, and detection algorithms for wideband spectrum sensing [J]. IEEE Transactions on Signal Processing, 2014, 62(19): 5107-5118.

[3] Taherpour, A., Gazor S., Nasiri-Kenari M. Invariant wideband spectrum sensing under unknown variances [J]. IEEE Transactions on Wireless Communications, 2009, 8(5), pp. 2182C2186.

[4] Tian, Z., Giannakis G. B. A wavelet approach to wideband spectrum sensing for cognitive radios [C]. $20061^{\text {st }}$ International Conference on Cognitive Radio Oriented Wireless Networks and Communications, 2006, pp. 1-5.

[5] Quan, Z., Cui S., Sayed A. H., et al. Optimal multiband joint detection for spectrum sensing in cognitive radio networks [J]. IEEE Transactions on Signal Process, 2009, 57(3): 1128-1140.

[6] Tian Z., Giannakis G. B. Compressive Sensing for Wideband Cognitive Radios [C]. IEEE International Conference on Acoustics, Speech, and Signal Processing, 2007, pp. 1357C60.

[7] Yu Z. Z., Chen X., Hoyos S., et al. Mixed-signal parallel compressive spectrum sensing for cognitive radios [J]. International Journal of Digital Multimedia Broadcasting, 2010: 1-10.

[8] Zeng F. Z., Li C., Tian Z. Distributed compressive spectrum sensing in cooperative multihop cognitive networks [J]. IEEE Journal of Selected Topics in Signal Processing, 2011, 5(1): 37-48.

[9] Zhang Z. H., Han Z., Li H. S., et al. Belief propagation based cooperative compressed spectrum sensing in wideband cognitive radio networks [J]. IEEE Transactions on Wireless Communications, 2011, 10(9): 3020-3031.

[10] Qing H. B., Liu Y. N., Xie G., Gao J. C. Wideband Spectrum Sensing for Cognitive Radios: A Multistage Wiener Filter Perspective [J]. IEEE Signal Processing Letters, 2015, 22(3): 332-335. 